\title{
Aspirin and clopidogrel hyporesponsiveness and nonresponsiveness in patients with coronary artery stenting
}

This article was published in the following Dove Press journal:

Vascular Health and Risk Management

6 November 2009

Number of times this article has been viewed

\author{
Rakesh K Sharma' \\ Hanumanth K Reddy' \\ Vibhuti N Singh ${ }^{2}$ \\ Rohit Sharma' \\ Donald J Voelker' \\ Girish Bhatt \\ 'Medical Center of South Arkansas, \\ El Dorado, University of Arkansas \\ for Medical Sciences, Little Rock. \\ AR, USA; ${ }^{2}$ Bayfront Medical Center, \\ St. Petersburg, University of South \\ Florida, Tampa, FL, USA
}

\begin{abstract}
Patients undergoing coronary artery stenting receive an antiplatelet regimen to reduce the risk of antithrombotic complications. Current guidelines recommend the use of acetyl salicylic acid (aspirin) and clopidogrel as evidenced by large clinical trials. There has been a concern about variable responses of patients to aspirin and clopidogrel which may predispose them to subacute stent thrombosis or late stent thrombosis. Up to $25 \%$ of patients with acute myocardial infarction (AMI) undergoing percutaneous coronary intervention (PCI) were found to have hyporesponsiveness or resistance to clopidogrel which may predispose them to recurrent events. Dual antiplatelet regimen is a standard therapy in these patients and there is always a concern about variable responses to aspirin and clopidogrel predisposing them to acute coronary syndrome (ACS). Prevalence of this hyporesponsiveness or resistance may be due to noncompliance, genetic mutations, co-morbid situations and concomitant use of other drugs. This issue is of considerable importance in the era of coronary drug eluting stents when a long-term dual antiplatelet regimen is needed. This paper is a review for clinicians taking care of such patients with hyporesponsiveness or nonresponsiveness to dual antiplatelet regimen.
\end{abstract}

Keywords: aspirin, clopidogrel, acute coronary syndrome, coronary artery stenting

\section{Background}

Do we manage hypertension without monitoring blood pressure? Do we manage diabetes mellitus (DM) without monitoring glucose? Do we manage warfarin sodium $\left(\right.$ Coumadin $^{\circledR}$ ) without monitoring international normalized ratio (INR)? If so, why do we use an antiplatelet regimen without monitoring platelet function testing. It is not uncommon for patients with recent coronary intervention with bare metal stents (BMS) or drug eluting stents (DES) to present to the emergency department with chest pain. Just as we monitor DM with a gluco-meter or warfarin with INR, we should monitor antiplatelet regimens in patients with coronary artery stenting. It is crucial to understand the heterogeneous response of patients to aspirin and clopidogrel which can result in unstable angina, or myocardial infarction (MI). ${ }^{1}$ Trials are ongoing (Gauging Responsiveness with a VerifyNow Assay-Impact on Thrombosis And Safety ${ }^{2}$ [GRAVITAS] and the ARCTIC study ${ }^{3}$ ) to determine whether tailored antiplatelet therapy, using platelet function testing, reduces major adverse cardiovascular events after DES.

Dual antiplatelet regimen with aspirin and clopidogrel has been shown to significantly reduce the cardiovascular events. ${ }^{4}$ Although clopidogrel mostly reduces risk of cardiovascular events after coronary stenting, a significant number of events still occur in these patients. ${ }^{1}$ These events may be due to subtherapeutic responses of some patients to aspirin and clopidogrel, predisposing them to subacute stent thrombosis or 
late stent thrombosis. Up to $25 \%$ of patients with acute $\mathrm{MI}$ (AMI) undergoing percutaneous coronary intervention (PCI) were found to have variable response to clopidogrel, predisposing them to recurrent events of acute coronary syndrome (ACS). ${ }^{1}$ Prevalence of this hyporesponsiveness or resistance may vary in certain co-morbid situations as described below. This is a critical issue in the era of DES with need of longterm dual antiplatelet regimens.

\section{Variable responses to antiplatelet agents and their mechanism}

The exact definition of "resistance" to antiplatelet therapy on the basis of physiology does not exist. However, there is a significant prevalence of variable response to dual antiplatelet regimens similar to different responses to anti-hypertensive therapy or warfarin therapy. Therefore, it is imperative to understand this variable response or hyporesponsiveness to aspirin and clopidogrel in these patients. A clear definition of this response should be established and, based on this, one may be able to categorize patients as a responders, hyporesponders, nonresponders, or resistant and thus manage their therapeutic regimen accordingly.

The effect of aspirin is mediated by the irreversible inactivation of cyclo-oxygenase (COX-1), leading to the prevention of thormboxane $\mathrm{A}_{2}$ generation from arachidonic acid. ${ }^{5}$ Following oral administration, aspirin is effective as an antiplatelet agent within 60 minutes. COX-1 is rapidly resynthesized by nucleated cells, such as endothelial cells, and therefore the effect of aspirin on nucleated cells lasts only for a relatively short time. In contrast, the effect of aspirin on platelets (anucleate cells) lasts for the life of platelets (7-10 days). Thienopyridines inhibit platelets via a blockade of P2Y12 adenosine diphosphate (ADP) receptors. ${ }^{6}$ As the mechanisms of aspirin and thienopyridines are complementary, the combination of both drugs has a greater degree of inhibition of platelet aggregation than either one alone. Clopidogrel is a pro-drug and is activated through sequential oxidative steps in the liver. After absorption, 85\% of drug is inactivated by esterases (hydrolase enzyme) and only $15 \%$ remains for activation by the liver to its active metabolite through several cytochrome 450 (CYP-450) proteins. Therefore, genetic mutation or polymorphism of such subenzymes can affect the therapeutic response of clopidogrel..$^{7-9}$

Based on this genetic polymorphism, by means of a simple buccal swab or blood sample, 3 phenotypes can be identified as being a: poor metabolizer (PM); intermediate metabolizer (IM), or normal metabolizer (NM). Underlying genetic mutation is due to variation in alleles (nucleotide sequences). The laboratory testing for this CYP-450 2C19 DNA mutation is done by identifying 8 different kinds of alleles. The nomenclature of this CYP 2C19 mutation is reported as $* 1$ (star 1$)$ to $* 8$ (star 8$).{ }^{10}$ The presence of CYP 2C $19 * 2$ allele is associated with reduced clopidogrel responsiveness and this is related to increased risk of MI, stent thrombosis. The frequency of this mutation in Caucasians and African Americans is $30 \% .{ }^{8}$ A poor metabolizer, as diagnosed by genetic testing, may exhibit different responsiveness to clopidogrel due to a failure to generate sufficient active form metabolites, which then leads to a lack of therapeutic effect. In a recent study, 162 healthy subjects were treated with clopidogrel and approximately 30\% of these were found to have at least one CYP2C19 reducedfunction allele that led to a relative reduction of $32.4 \%$ of the active metabolite of clopidogrel in plasma. ${ }^{8}$ Moreover, among subjects treated with clopidogrel in TRITON-TIMI 38 , carriers of the $* 2$ defective gene (CYP2C19 reducing function allele called $* 2$ genotype) had a relative increase of $53 \%$ in the composite primary efficacy outcome of the risk of death from cardiovascular causes, MI, or stroke compared to noncarriers. ${ }^{11}$ This may lead to the need for unconventional doses of clopidogrel or an alternative oral antiplatelet drug in such patients. CYP2C19 metabolizing enzyme also catalyzes the biotransformation of many other drugs ${ }^{12}$ and the concomitant use of such drugs with clopidogrel may also change the efficacy of antiplatelet therapy. Various biological factors such as genetic polymorphism or gene mutation may account for such hyporesponsiveness or nonresponsiveness. Other causes of hyporesponsiveness of antiplatelet drugs may be as simple as noncompliance ${ }^{13-16}$ or poor absorption, ${ }^{17}$ due to abnormalities in the mechanism of action or genetic makeup. ${ }^{18}$ Smoking has also been proposed to cause hyporesponsiveness in both aspirin and clopidogrel patients, ${ }^{1,16,19}$ whereas other researchers have reported that smokers were likely to be

Table I Mechanisms of "resistance" to aspirin and clopidogrel

Non compliance

Poor absorption

Sub optimal dose

Smoking

Genetic polymorphism

Thrombocytosis

Concomitant medication

Co-morbid conditions

Severe coronary artery disease 
responders. ${ }^{1}$ Gremmel and associates reported that cigarette smoking was associated with enhanced efficacy of clopidogrel but not aspirin, on platelet inhibition in vitro testing. ${ }^{20}$ Bliden et al have also shown that current smokers prescribed clopidogrel showed lower platelet aggregation. ${ }^{21}$

\section{Methodologies for the evaluation of platelet function}

There are many different types of platelet function tests, which vary in methodological complexity. Different methods of evaluating platelet function revolve around the measurement of platelet aggregation, platelet reactivity, platelet receptor expression, measurement of platelet-released factors on activation, and intracellular platelet signaling. Although there is no standard definition of hyporesponsiveness or nonresponsiveness or resistance to antiplatelet drugs, it is important to identify the patients with these conditions. In the published literature, definitions of aspirin resistance or clopidogrel resistance depend upon the different methodologies used in studies (Table 2). A brief description of the most commonly used tests to evaluate the efficacy of antiplatelet drugs are described as follows.

\section{PFA-100 system}

The PFA-100 system simulates high shear platelet function within the test cartridges. ${ }^{22}$ It is a simple, rapid test and only a small sample of blood is required. PFA-100 tests use agonists such as epinephrine or ADP to mimic high shear stress on platelets. This test reports the platelet function as "closure time" (CT), which is the time platelets take to occlude an aperture in the membrane coated with collagen/epinephrine (C/EPI) or collagen/ADP (C/ADP). Because clopidogrel does not affect $\mathrm{CT}$ with $\mathrm{C} / \mathrm{EPI}$, the $\mathrm{C} / \mathrm{ADP}$ test is used for clopidogrel efficacy. ${ }^{23}$ This test has not been shown to have significant correlation with other assays like light transmittance aggregometry (LTA), vasodilator-stimulated phosphoprotein (VASP) or VerifyNow ${ }^{\circledR} \cdot{ }^{15,24}$

\section{Platelet-platelet aggregation testing}

Aggregation is the most common measure of platelet reactivity and platelet inhibition. This assay is based on platelet aggregation by stimulation with various agonists. Such aggregation testing between pretreatment and post-treatment of aspirin or clopidogrel are the most common estimates of responsiveness to aspirin or a P2Y12 receptor inhibitor like clopidogrel. ${ }^{25}$ Historically, the "gold standard" test is LTA. ${ }^{17}$ LTA uses an optical device that measures the rate and extent of change in light transmittance caused by platelets aggregating in a whole blood sample. This test determines the level of platelet function in response to a variety of agonists, as there are many pathways through which antiplatelet medications work. Blood samples with inhibited platelets (with antiplatelet medication) will produce low levels of light transmittance compared to normal functioning platelets which aggregate normally. This test has been widely used to measure the effect of dual antiplatelet regimens. LTA testing is tedious, time consuming and requires specialized staff, compared to rapid point-of-care (POC) assays, which are simple, less laborious and time efficient. VerifyNow ${ }^{\circledR}$ is one of the POC platelet aggregation tests that does not require sample preparation or pipetting and is also strongly correlated with LTA. ${ }^{26,24}$ By this methodology, aspirin response is reported as aspirin reaction units (ARU). Aspirin blocks platelet activation by preventing COX-1 enzyme from converting arachidonic acid to thormboxane A2. The extent of this blockade is determined as ARUs. More than 550 ARUs is considered as diminished aspirin-induced platelet dysfunction. ${ }^{27-30}$ Thienopyridines, like clopidogrel, block platelet activation via P2Y12 ADP receptors and the extent of this blockade is reported as P2Y12 reaction units (PRU). This test takes advantage of different receptors of platelets stimulated by different agonists. Thrombin receptors are strong platelet activators and function independently of P2Y12 ADP receptors. The base value (base PRU) is calculated by stimulating these receptors to estimate the total possible platelet aggregation irrespective

Table 2 Definition of aspirin and clopidogrel hyporesponsiveness, nonresponsiveness or "resistance"

\begin{tabular}{|c|c|c|c|c|c|}
\hline \multicolumn{2}{|l|}{ Aspirin } & \multicolumn{4}{|l|}{ Clopidogrel $^{\mathrm{a}}$} \\
\hline & Reference & Responders & Hyporesponder & Resistant & Reference \\
\hline ARU $>550$ (lack of ASA-induced platelet dysfunction) & 39 & $>30 \%$ & $<10 \%-29 \%$ & $<10 \%$ & 28 \\
\hline C/EPI-CT $<193$ seconds & 14 & $>40 \%$ & & $<40 \%$ & 27 \\
\hline \multirow[t]{2}{*}{ C/ADP-CT $<121$ seconds } & 13 & $>30 \%$ & $<10 \%-30 \%$ & $10 \%$ & 29 \\
\hline & & $>10 \%$ & & $<10 \%$ & 30 \\
\hline
\end{tabular}

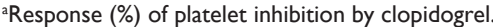

Abbreviations: $\mathrm{ARU}$, aspirin reaction units; $\mathrm{C} / \mathrm{EPI}-\mathrm{CT}$, collagen/epinephrine closure time; C/ADP-CT, collagen/ADP closure time; $\mathrm{ASA}$, aspirin. 
of the patient taking or not taking clopidogrel. Then the extent of platelet inhibition by clopidogrel can be measured by using a selective ADP agonist to measure P2Y12 ADP receptor inhibition. The percentage inhibition of $\mathrm{P} 2 \mathrm{Y} 12$ ADP receptors is determined by the difference between base PRU and PRU determined from ADP agonist (as illustrated in Figure 1). Less than $20 \%$ inhibition indicates low to no clopidogrel-induced inhibition of platelet function.

\section{VASP phosphorylation}

The surface expression of platelets can be determined by flow cytometery. Using monoclonal antibodies, platelet function can be determined by exploiting the different receptor expression of resting and activated platelets. ${ }^{17}$ This test (VASP) exploits the mechanism of intracellular signaling; ${ }^{24,31}$ the advantage of VASP phosphorylation testing is its specificity for the P2Y12 signaling pathway, although drawbacks include sample preparation, the need for experienced staff, and the expenses of the process.

\section{Tests dependent on factors released from activated platelets}

Some tests measure the factors released by activated platelets as a measure of platelet activation such as serum or thormboxane $\mathrm{B}_{2}$ and urinary 11-dehydro-thromboxane $\mathrm{B}_{2} \cdot{ }^{17}$ These are COX-dependent and nonspecific. Moreover, urinary 11-dehydro-thromboxane depends on renal function.
Another test, platelet-derived-miroparticles is expensive, and requires sample preparation and flow cytometery. ${ }^{26}$

\section{Factors causing hyporesponsiveness or nonresponsiveness}

A major concern after interventional procedures is an ischemic event, often caused by activated platelets at the site of a coronary stent implantation, which underscores the critical role of antiplatelet agents in PCI. Most of the time, the assumption is that dual antiplatelet regimens are efficacious without any objective testing. Unfortunately, not all patients respond equally and up to one-third of patients on an antiplatelet regimen do not experience the expected results. ${ }^{26}$ It is very important to identify these nonresponders, especially with the increasing use of DES. Nonresponders are at 5 times greater risk of MI, stent thrombosis and death than responders. ${ }^{32,33}$ Numerous factors may contribute to this unresponsiveness which may include noncompliance, drug interactions, DM, chronic renal failure or genetic makeup.

\section{Factors modifying the efficacy of aspirin}

Recent observations suggest that the primary cause for aspirin resistance may be poor compliance. ${ }^{34,35}$ Hence, it is imperative to ensure compliance with an aspirin regimen prior to platelet function testing. Another important factor leading to aspirin resistance may be concomitant use of
Thienopyridines block platelet activation via $\mathrm{P}_{2} \mathrm{Y}_{12}$ ADP receptor PRU value is determined from the extent of this blockade

Thrombin receptors activate platelets independently of $\mathrm{P}_{2} \mathrm{Y}_{12}$

The BASE result is detemined by stimulating these receptors to estimate total possible platelet aggregation while patient is on clopidogrel

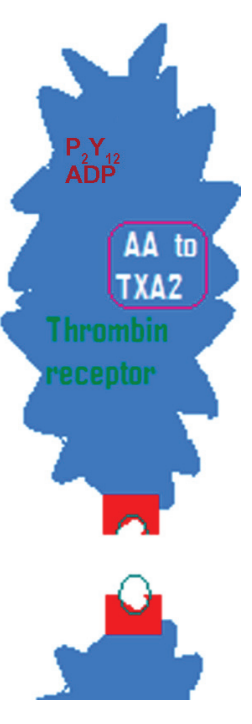

Aspirin blocks platelet activation by preventing COX-1 enzyme by preventing conversion of arachidonic acid to thromboxane $A_{2}$

ARU: represents extent of this blockade

$\%$ inhibition is determined by the $\%$ difference between PRU and BASE

Figure I Understanding platelet function testing. Abbreviations: $A A$, arachidonic acid; PRU, P2YI 2 reaction units; $A R U$, aspirin reaction units; TXA2, thromboxane $A_{2}$. 
nonsteroidal anti-inflammatory drugs such as ibuprofen, which interferes with COX-1 acetylation. Even though some investigators have suggested increasing the aspirin dose in such patients, in vitro studies have shown that increasing the dose of aspirin may not affect COX-1 inhibition ${ }^{36}$ and may work through a non-COX-1 inhibition pathway. Aspirin resistance or hyporesponsiveness may be also due to age or gender issues. Many studies have shown that women are more likely to have aspirin resistance and the majority of these subjects are postmenopausal. ${ }^{16,37}$ However, this has been disputed in other studies. ${ }^{38}$ Some studies have shown that advanced age may be an important factor ${ }^{39}$ and may be due to decreased metabolism of aspirin in old age. Therefore, it is important to test the antiplatelet efficacy of aspirin in patients with PCI.

Furthermore, this may help determine if baby aspirin is useful in such patients. Certain co-morbid conditions such as DM and chronic renal failure may cause hyporesponsiveness to aspirin. ${ }^{14,39,40}$ Some studies found a higher prevalence of nonresponders in smokers while other researchers have disputed this. ${ }^{41}$

\section{Factors modifying the efficacy of clopidogrel}

The most important cause of variable platelet activity suppression may be noncompliance and inadequate dosing. Resistance to clopidogrel may co-exist with aspirin and this may be prevalent up to $50 \%$ of patients with aspirin resistance ${ }^{40}$ (or it may present by itself). Subacute thrombosis of coronary stents in the CREST study was found to be more common in patients with hyporesponsiveness to clopidogrel. ${ }^{42}$ Clopidogrel is a pro-drug and its active metabolite irreversibly inhibits the binding of P2Y12 ADP receptor on platelets. ${ }^{43}$ Such metabolism invokes the possibility of the concurrent use of other medications as contributing factors to hyporesponsiveness in patients with coronary artery stenting. Concomitant use of other drugs, such as calcium channel blockers (CCB), angiotensin-converting enzyme (ACE) inhibitors, or beta blockers, does not cause hyporesponsiveness to clopidogrel, although a higher number of nonresponders is seen with concomitant use of $\mathrm{CCB}$ and $\mathrm{ACE}$ inhibitors. ${ }^{30} \mathrm{~A}$ recent study by Siller-Matula et al showed that coadministration of CCB decreased platelet inhibition ${ }^{44}$ though in vitro incubation with CCB did not alter platelet aggregation in patients taking clopidogrel. This finding suggests that the negative effect in vivo may be at the level of metabolic pathways.

There has been much concern about the use of proton pump inhibitors (PPI) in conjunction with clopidogrel.
A recent retrospective review of 8205 patients published in JAMA showed a strong association of adverse clinical outcomes when clopidogrel was used in conjunction with omeprazole, ${ }^{45}$ though this was recently disputed in a late breaking trial (COGENT) at the TCT Conference on September 24, 2009 in San Francisco, USA (http://www. tctmd.com/Show.aspx?id=85972). Omeprazole $\left(\operatorname{Losec}^{\circledR}\right.$ or Prilosec $^{\circledR}$ ) is both a substrate and an inhibitor of CYP 2C19 and it may decrease the metabolism of clopidogrel to its active metabolite. Other PPIs that are CYP2c19 inhibitors are esomeprazole $\left(\right.$ Nexium $\left.^{\circledR}\right)$, lansoprazole $\left(\right.$ Prevacid $\left.^{\circledR}\right)$ and rabeprazole $\left(\right.$ Aciphex $\left.^{\circledR}\right)$. A recent clopidogrel medical outcome study has also suggested a similar interaction between PPI and clopidogrel. ${ }^{46}$

\section{Management strategies}

A standard definition of hyporesponsiveness or nonresponsiveness or resistance does not exist. One may also argue that incidence of resistance or hyporesponsiveness is overestimated. But there are several studies showing that a poor response to these drugs may translate into adverse outcomes as discussed earlier. Increasing the dose of aspirin has been shown to improve the response in some patients. ${ }^{39}$ The impact of increased clopidogrel dosing was evaluated in patients with suboptimal response to clopidogrel in the OPTIMUS trial. ${ }^{47}$ This study showed that a dose of $150 \mathrm{mg}$ of clopidogrel significantly decreased the platelet aggregation compared to a $75 \mathrm{mg}$ dose. Likewise, Gurbel et al ${ }^{48,49}$ showed better efficacy of $600 \mathrm{mg}$ compared with $300 \mathrm{mg}$ loading dose. Furthermore, the combination of clopidogrel with a synergistic antiplatelet agent like dipyridamole can also improve the response..$^{50}$ Theoretically cytochrome $\mathrm{P} 450$ inducers can increase the active metabolites of clopidogrel, which could be an alternative to an increased dose, especially if there is gastrointestinal intolerance to the drug.

\section{New drugs on the horizon}

Other drugs on the horizon include a new thienopyridines agent (prasugrel) which has been evaluated in several large scale trials. ${ }^{51,52}$ In these studies, a loading dose of $60 \mathrm{mg}$ and maintenance dose of $10 \mathrm{mg}$ of prasugrel produced more consistent platelet inhibition compared to clopidogrel with a loading of $300 \mathrm{mg}$ and maintenance of $75 \mathrm{mg}$. This translated into a lower rate of combined primary outcomes of death, nonfatal MI and stroke in high-risk patients with ACS. Prasugrel also demonstrated a 50\% reduction in stent thrombosis in one trial. ${ }^{11}$ However, superior efficacy of 
this agent has been somewhat offset by the increased risk of bleeding. While this drug has been approved in Europe for some time, the Federal Drug Administration (FDA) approval of this antiplatelet drug on July 10, 2009 was a major step forward in patients with ACS and PCI in the US. This may also be helpful in patients with CYP2C19 mutation, as it may not affect the pharmacokinetics and pharmacodynamics of prasugrel compared to clopidogrel.

Another highly selective, oral, nonthienopyridine drug is ticagrelor (AZD6140) which has been investigated in a large phase III trial. ${ }^{53,54}$ This also works by antagonizing ADP at the P2Y12 receptors; it does not require transformation to active metabolite and has a half-life of 7 to 8 hours. ${ }^{55}$ The safety and efficacy of ticagrelor were investigated in a trial named PLATO (Platelet Inhibition and Patient Outcome), the results of which were presented at European Society of Cardiology (ESC 2009) and simultaneously published in $N$ Engl $\mathrm{J} \mathrm{Med} .{ }^{56}$ In this study patients were randomized for a double blind trial to a Ticagrelor group (9333 patients with loading dose of $180 \mathrm{mg}$ followed by $90 \mathrm{mg}$ twice daily) or a clopidogrel group (9291 patients with loading dose $300 \mathrm{mg}$ followed by $75 \mathrm{mg}$ daily). ${ }^{56}$ Death from vascular causes, MI or stroke within 12 months occurred less frequently in the ticagrelor group. The potential availability of 3 ADP receptor P2Y12 inhibitors may make it possible to individualize antiplatelet regimens rather than a "one size fits all strategy". Ticagrelor may be preferred in acute ACS patients with unknown anatomy, in whom coronary artery bypass grafting may be anticipated, as this is a reversible ADP receptor inhibitor. There may be a potential for switching clopidogrel or prasugrel to ticagrelor in patients who need elective surgery. This may become the antiplatelet agent of choice in situations where surgical procedures cannot be deferred. ${ }^{55}$ Other new classes of antiplatelet agents include thrombin receptor antagonists called protease-activated receptor (PAR-1) inhibitors. ${ }^{57,58}$ PAR-1 is the main platelet receptor for thrombin, the inhibition of which may lead to the development of novel antiplatelet agents.

\section{Conclusions}

Platelets display an enormous complexity by their variety of receptors and the myriad of molecules they secrete. These receptors and molecules mediate a large number of physiologic and pathophysiologic processes and hence are a target for multiple antiplatelet agents. Variable responses to oral antiplatelet regimens are well known. Therefore, it is important to distinguish between hyporesponsiveness or nonresponsiveness or resistance (failure to inhibit platelets activity), and treatment failure (the clinical outcome of a recurrence of ischemic events). As described earlier, the prevalence of hyporesponsiveness or nonresponsiveness or resistance may be an aberration of the methodology; however, there is clearly accumulating evidence that in vivo resistance to oral antiplatelet regimens leads to a higher risk of atherothrombotic complications such as unstable angina, and MI. New developments in drugs may offer a narrow range of response variability leading to more predictive efficacy. Antiplatelet testing or genotyping may help uncover the underlying mechanisms of hyporesponsiveness or nonresponsiveness or resistance and help the development of personalized patient oral antiplatelet regimens. There is a need for large-scale studies documenting the efficacy of point of care assessment of platelet function, which will be a true departure from the "one size fits all" strategy in managing antiplatelet regimens in coronary artery stenting.

\section{Disclosures}

The authors declares no conflicts of interest.

\section{References}

1. Matetzky S, Shenkman B, Guetta V, et al. Clopidogrel resistance is associated with increased risk of recurrent atherothrombotic events in patients with acute myocardial infarction. Circulation. 2004;109(25):3171-3175.

2. GRAVITAS: Gauging responsiveness with a verifynow assay-impact on thrombosis and safety in patients with acute myocardial infarction. http://clinicaltrails.gov/ct2/show/NCT00645918.

3. Double randomization of montoring adjusted anti-platelet treatment versus a common anti-platelet treatment for DES Implantation, and interuption versus continuation of double antiplatelet therapy. URL http://clinicaltrials.gov/archive/NCT00827411/2009_01_21.2009.

4. A randomised, blinded, trial of clopidogrel versus aspirin in patients at risk of ischaemic events (CAPRIE). CAPRIE Steering Committee. Lancet. 1996;348(9038):1329-1339.

5. Patrono C. Aspirin as an antiplatelet drug. $N$ Engl J Med. 1994;330(18):1287-1294.

6. Kunapuli SP, Dorsam RT, Kim S, Quinton TM. Platelet purinergic receptors. Curr Opin Pharmacol. 2003;3(2):175-180.

7. Simon T, Verstuyft C, Mary-Krause M, et al. Genetic determinants of response to clopidogrel and cardiovascular events. $N$ Engl J Med. 2009;360(4):363-375.

8. Mega JL, Close SL, Wiviott SD, et al. Cytochrome $\mathrm{p}-450$ polymorphisms and response to clopidogrel. N Engl J Med. 2009;360(4):354-362.

9. Suh JW, Koo BK, Zhang SY, et al. Increased risk of atherothrombotic events associated with cytochrome $\mathrm{P} 4503 \mathrm{~A} 5$ polymorphism in patients taking clopidogrel. CMAJ. 2006;174(12):1715-1722.

10. Blaisdell J, Mohrenweiser H, Jackson J, et al. Identification and functional characterization of new potentially defective alleles of human CYP2C19. Pharmacogenetics. 2002;12(9):703-711.

11. Wiviott $\mathrm{SD}$, Braunwald $\mathrm{E}, \mathrm{McCabe} \mathrm{CH}$, et al. Intensive oral antiplatelet therapy for reduction of ischaemic events including stent thrombosis in patients with acute coronary syndromes treated with percutaneous coronary intervention and stenting in the TRITON-TIMI 38 trial: a subanalysis of a randomised trial. Lancet. 2008;371(9621):1353-1363. 
12. Meisel C, Roots I, Cascorbi I, Brinkmann U, Brockmoller J. How to manage individualized drug therapy: application of pharmacogenetic knowledge of drug metabolism and transport. Clin Chem Lab Med. 2000;38(9):869-876.

13. Christiaens L, Macchi L, Herpin D, et al. Resistance to aspirin in vitro at rest and during exercise in patients with angiographically proven coronary artery disease. Thromb Res. 2002;108(2-3):115-119.

14. Angiolillo DJ, Fernandez-Ortiz A, Bernardo E, et al. Influence of aspirin resistance on platelet function profiles in patients on long-term aspirin and clopidogrel after percutaneous coronary intervention. Am J Cardiol. 2006;97(1):38-43.

15. Gum PA, Kottke-Marchant K, Welsh PA, White J, Topol EJ. A prospective, blinded determination of the natural history of aspirin resistance among stable patients with cardiovascular disease. $\mathrm{J} \mathrm{Am} \mathrm{Coll}$ Cardiol. 2003;41(6):961-965.

16. Gum PA, Kottke-Marchant K, Poggio ED, et al. Profile and prevalence of aspirin resistance in patients with cardiovascular disease. $\mathrm{Am} \mathrm{J}$ Cardiol. 2001;88(3):230-235.

17. Michelson AD. Platelet function testing in cardiovascular diseases. Circulation. 2004;110(19):e489-e493.

18. Awtry EH, Loscalzo J. Aspirin. Circulation. 2000;101(10):1206-1218.

19. Ferguson AD, Dokainish H, Lakkis N. Aspirin and clopidogrel response variability: review of the published literature. Tex Heart Inst J. 2008;35(3):313-320.

20. Gremmel T, Steiner S, Seidinger D, Koppensteiner R, Panzer S, Kopp CW. Smoking promotes clopidogrel-mediated platelet inhibition in patients receiving dual antiplatelet therapy. Thromb Res. 2009;124(5):588-591.

21. Bliden KP, Dichiara J, Lawal L, et al. The association of cigarette smoking with enhanced platelet inhibition by clopidogrel. J Am Coll Cardiol. 2008;52(7):531-533.

22. Favaloro EJ. Clinical application of the PFA-100. Curr Opin Hematol. 2002;9(5):407-415.

23. Fontana P, Nolli S, Reber G, de Moerloose P. Biological effects of aspirin and clopidogrel in a randomized cross-over study in 96 healthy volunteers. J Thromb Haemost. 2006;4(4):813-819.

24. Paniccia R, Antonucci E, Gori AM, et al. Different methodologies for evaluating the effect of clopidogrel on platelet function in high-risk coronary artery disease patients. J Thromb Haemost. 2007;5(9):1839-1847.

25. Smith JW, Steinhubl SR, Lincoff AM, et al. Rapid platelet-function assay: an automated and quantitative cartridge-based method. Circulation. 1999;99(5):620-625.

26. Gurbel PA, Becker RC, Mann KG, Steinhubl SR, Michelson AD. Platelet function monitoring in patients with coronary artery disease. J Am Coll Cardiol. 2007;50(19):1822-1834.

27. Angiolillo DJ, Fernandez-Ortiz A, Bernardo E, et al. Identification of low responders to a 300-mg clopidogrel loading dose in patients undergoing coronary stenting. Thromb Res. 2005;115(1-2): 101-108.

28. Muller I, Besta F, Schulz C, Massberg S, Schonig A, Gawaz M. Prevalence of clopidogrel non-responders among patients with stable angina pectoris scheduled for elective coronary stent placement. Thromb Haemost. 2003;89(5):783-787.

29. Samara WM, Bliden KP, Tantry US, Gurbel PA. The difference between clopidogrel responsiveness and posttreatment platelet reactivity. Thromb Res. 2005;115(1-2):89-94.

30. Gurbel PA, Bliden KP, Hiatt BL, O'Connor CM. Clopidogrel for coronary stenting: response variability, drug resistance, and the effect of pretreatment platelet reactivity. Circulation. 2003;107(23):2908-2913.

31. Geiger J, Brich J, Honig-Liedl P, et al. Specific impairment of human platelet P2Y(AC) ADP receptor-mediated signaling by the antiplatelet drug clopidogrel. Arterioscler Thromb Vasc Biol. 1999;19(8):2007-2011.

32. Buonamici P, Marcucci R, Migliorini A, et al. Impact of platelet reactivity after clopidogrel administration on drug-eluting stent thrombosis. J Am Coll Cardiol. 2007;49(24):2312-2317.
33. Price MJ, Endemann S, Gollapudi RR, et al. Prognostic significance of post-clopidogrel platelet reactivity assessed by a point-of-care assay on thrombotic events after drug-eluting stent implantation. Eur Heart J. 2008;29(8):992-1000.

34. Tantry US, Bliden KP, Gurbel PA. Overestimation of platelet aspirin resistance detection by thrombelastograph platelet mapping and validation by conventional aggregometry using arachidonic acid stimulation. J Am Coll Cardiol. 2005;46(9):1705-1709.

35. Michelson AD, Cattaneo M, Eikelboom JW, et al. Aspirin resistance: position paper of the Working Group on Aspirin Resistance. J Thromb Haemost. 2005;3(6):1309-1311.

36. Patrono C, Coller B, Dalen JE, et al. Platelet-active drugs: the relationships among dose, effectiveness, and side effects. Chest. 2001;119(1 Suppl):39S-63S.

37. Chen WH, Lee PY, Ng W, Tse HF, Lau CP. Aspirin resistance is associated with a high incidence of myonecrosis after non-urgent percutaneous coronary intervention despite clopidogrel pretreatment. J Am Coll Cardiol. 2004;43(6):1122-1126.

38. Wang JC, Aucoin-Barry D, Manuelian D, et al. Incidence of aspirin nonresponsiveness using the Ultegra Rapid Platelet Function AssayASA. Am J Cardiol. 2003;92(12):1492-1494.

39. Lee PY, Chen WH, Ng W, et al. Low-dose aspirin increases aspirin resistance in patients with coronary artery disease. $\mathrm{Am} \mathrm{J} \mathrm{Med.}$ 2005;118(7):723-727.

40. Lev EI, Patel RT, Maresh KJ, et al. Aspirin and clopidogrel drug response in patients undergoing percutaneous coronary intervention: the role of dual drug resistance. J Am Coll Cardiol. 2006;47(1):27-33.

41. Macchi L, Christiaens L, Brabant S, et al. Resistance to aspirin in vitro is associated with increased platelet sensitivity to adenosine diphosphate. Thromb Res. 2002;107(1-2):45-49.

42. Gurbel PA, Bliden KP, Samara W, et al. Clopidogrel effect on platelet reactivity in patients with stent thrombosis: results of the CREST Study. J Am Coll Cardiol. 2005;46(10):1827-1832.

43. Labarthe B, Theroux P, Angioi M, Ghitescu M. Matching the evaluation of the clinical efficacy of clopidogrel to platelet function tests relevant to the biological properties of the drug. J Am Coll Cardiol. 2005;46(4):638-645.

44. Siller-Matula J, Schror K, Wojta J, Huber K. Thienopyridines in cardiovascular disease: focus on clopidogrel resistance. Thromb Haemost. 2007;97(3):385-393.

45. Ho PM, Maddox TM, Wang L, et al. Risk of adverse outcomes associated with concomitant use of clopidogrel and proton pump inhibitors following acute coronary syndrome. JAMA. 2009;301(9):937-944.

46. Aubert RE. Proton Pump Inhibitors Effect on Clopidogrel Effectiveness: The Clopidogrel Medco Outcome Study. Circulation. 2008;(118:S):815.

47. Angiolillo DJ, Shoemaker SB, Desai B, et al. Randomized comparison of a high clopidogrel maintenance dose in patients with diabetes mellitus and coronary artery disease: results of the Optimizing Antiplatelet Therapy in Diabetes Mellitus (OPTIMUS) study. Circulation. 2007;115(6):708-716.

48. Muller I, Seyfarth M, Rudiger S, et al. Effect of a high loading dose of clopidogrel on platelet function in patients undergoing coronary stent placement. Heart. 2001;85(1):92-93.

49. Gurbel PA, Bliden KP, Hayes KM, Yoho JA, Herzog WR, Tantry US The relation of dosing to clopidogrel responsiveness and the incidence of high post-treatment platelet aggregation in patients undergoing coronary stenting. J Am Coll Cardiol. 2005;45(9):1392-1396.

50. Serebruany VL, Malinin AI, Sane DC, et al. Magnitude and time course of platelet inhibition with Aggrenox and Aspirin in patients after ischemic stroke: the AGgrenox versus Aspirin Therapy Evaluation (AGATE) trial. Eur J Pharmacol. 2004;499(3):315-324.

51. Wiviott SD, Antman EM, Gibson CM, et al. Evaluation of prasugrel compared with clopidogrel in patients with acute coronary syndromes: design and rationale for the TRial to assess Improvement in Therapeutic Outcomes by optimizing platelet InhibitioN with prasugrel Thrombolysis In Myocardial Infarction 38 (TRITON-TIMI 38). Am Heart J. 2006;152(4):627-635. 
52. Wiviott SD, Braunwald E, McCabe $\mathrm{CH}$, et al. Prasugrel versus clopidogrel in patients with acute coronary syndromes. $N$ Engl J Med. 2007;357(20):2001-2015.

53. Husted S, Emanuelsson H, Heptinstall S, Sandset PM, Wickens M, Peters G. Pharmacodynamics, pharmacokinetics, and safety of the oral reversible P2Y12 antagonist AZD6140 with aspirin in patients with atherosclerosis: a double-blind comparison to clopidogrel with aspirin. Eur Heart J. 2006;27(9):1038-1047.

54. Cannon CP, Husted S, Harrington RA, et al. Safety, tolerability, and initial efficacy of AZD6140, the first reversible oral adenosine diphosphate receptor antagonist, compared with clopidogrel, in patients with non-ST-segment elevation acute coronary syndrome: primary results of the DISPERSE-2 trial. J Am Coll Cardiol. 2007;50(19):1844-1851.
55. Schomig A. Ticagrelor - is there need for a new player in the antiplatelet-therapy field? N Engl J Med. 2009;361(11):1108-1111.

56. Wallentin L, Becker RC, Budaj A, et al. Ticagrelor versus clopidogrel in patients with acute coronary syndromes. $N$ Engl J Med. 2009; 361(11):1045-1057.

57. Jennings LK, EarhartA, Becker RC, Reyderman L, VeltriE, Harrington RA. Abstract 3010: Thrombin receptor antagonist (TRA; SCH530348) is a selective, potent inhibitor of PAR1 activity with predictable pharmacokinetics. Circulation. 2007;116(16 MeetingAbstracts): II674-.

58. Wu CC, Teng CM. Comparison of the effects of PAR1 antagonists, PAR4 antagonists, and their combinations on thrombin-induced human platelet activation. Eur J Pharmacol. 2006;546(1-3):142-147.

\section{Publish your work in this journal}

Vascular Health and Risk Management is an international, peerreviewed journal of therapeutics and risk management, focusing on concise rapid reporting of clinical studies on the processes involved in the maintenance of vascular health; the monitoring, prevention and treatment of vascular disease and its sequelae; and the involvement of metabolic disorders, particularly diabetes. This journal is indexed on PubMed Central and MedLine. The manuscript management system is completely online and includes a very quick and fair peer-review system, which is all easy to use. Visit http://www.dovepress.com/ testimonials.php to read real quotes from published authors. 